\title{
When Uniformly-continuous Implies Bounded
}

\author{
ANTHONY G. O'FARRELL ${ }^{1}$
}

\section{INTRODUCTION}

Let $(X, \rho)$ and $(Y, \sigma)$ be metric spaces. A function $f: X \rightarrow Y$ is (by definition) bounded if the image of $f$ has finite $\sigma$-diameter. It is well-known that if $X$ is compact then each continuous $f: X \rightarrow Y$ is bounded. Special circumstances may conspire to force all continuous $f: X \rightarrow Y$ to be bounded, without $Y$ being compact. For instance, if $Y$ is bounded, then that is enough. It is also enough that $X$ be connected and that each connected component of $Y$ be bounded. But if we ask that all continuous functions $f: X \rightarrow Y$, for arbitrary $Y$, be bounded, then this requires that $X$ be compact.

What about uniformly-continuous maps? Which $X$ have the property that each uniformly-continuous map from $X$ into any other metric space must be bounded?

We begin with an observation.

Lemma 1.1. Let $(X, \rho)$ be a metric space. Then the following are equivalent:

(1) Each uniformly-continuous map from $X$ into another metric space is bounded.

(2) Each uniformly-continuous map from $X$ into $\mathbb{R}$ (with the usual metric) is bounded.

Proof. Obviously (1) implies (2). The other direction follows from the facts that: (a) $f: X \rightarrow Y$ is bounded if and only if each (or any one) of the compositions

$$
\sigma(b, \bullet) \circ f: x \mapsto \sigma(b, f(x)) \quad(b \in Y)
$$

is bounded, and (b) the composition $\sigma(b, \bullet)$ is uniformly-continuous if $f$ is uniformly-continuous.

\footnotetext{
${ }^{1}$ Supported by EU Research Training Network Contract no. HPRN-CT-200000116.
} 
This allows us to concentrate on the case $Y=\mathbb{R}$, with the usual metric.

1.1. Example. Each uniformly-continuous function $f:(a, b) \rightarrow \mathbb{R}$, mapping a bounded open interval to $\mathbb{R}$, is bounded. Indeed, given such an $f$, choose $\delta>0$ with the property that the modulus of continuity $\omega_{f}(\delta)<1$, i.e.,

$$
|x-y|<\delta \Longrightarrow|f(x)-f(y)|<1 .
$$

Take $n \in \mathbb{N}$ greater than $(b-a) / \delta, h=(b-a) / n$, and $a_{i}=a+i h$ $(0 \leq i \leq n)$. Then

$$
|f(x)| \leq 1+\max \left\{\left|f\left(a_{i}\right)\right|: 1 \leq i \leq n-1\right\} .
$$

A very similar argument shows that if $X$ is totally-bounded, then each uniformly-continuous function from $X$ is bounded. However, this is not the whole story.

1.2. Example. Let $X$ be the unit ball of $\ell^{\infty}$, i.e., the space of all bounded sequences $\left\{a_{n}\right\}$ of complex numbers, with the metric induced by the supremum norm:

$$
\rho\left(\left\{a_{n}\right\},\left\{b_{n}\right\}\right)=\sup _{n}\left|a_{n}-b_{n}\right| .
$$

Suppose $f: X \rightarrow \mathbb{R}$ is uniformly-continuous, and choose $\delta>0$ such that $\omega_{f}(\delta)<1$. Let $m \in \mathbb{N}$ be the ceiling of $1 / \delta$. Then for each $a=\left\{a_{n}\right\} \in X$, taking $h=\sup _{n}\left|a_{n}\right| / m$ and $b_{i}=i h a$, we have

$$
|f(a)| \leq|f(0)|+\sum_{i=1}^{m}\left|f\left(b_{i}\right)-f\left(b_{i-1}\right)\right| \leq|f(0)|+m .
$$

Thus $f$ is bounded. However, $X$ is not totally-bounded.

\section{EPSILON-STEP TERRITORIES}

For $\varepsilon>0$ and $a, b \in X$, we say that $a$ is $\varepsilon$-step-equivalent to $b$ if there exist points $a_{0}=a, a_{1}, \ldots, a_{n}=b$, belonging to $X$, with $\rho\left(a_{i-1}, a_{i}\right) \leq \varepsilon$ for each $i$. This defines an equivalence relation on $X$ (for each fixed $\varepsilon>0$ ). We call the equivalence classes $\varepsilon$-step territories, and denote the territory of a point $a$ by $T_{\varepsilon}(a)$, or just $T(a)$, if the value of $\varepsilon$ is clear from the context.

For $\varepsilon>0$ and $a, b \in X$, we denote by $s_{\varepsilon}(a, b)$ (or just $s(a, b)$ ) the infimum of those $n \in \mathbb{N}$ (if any) for which there exist $a_{0}=a$, $a_{1}, \ldots, a_{n}=b$ belonging to $X$, with $\rho\left(a_{i-1}, a_{i}\right) \leq \varepsilon$. Obviously, $s(a, b)<+\infty$ if and only if $T(a)=T(b)$. 
We say that a territory $T(a)$ is $\varepsilon$-step-bounded if

$$
\sup _{x \in T(a)} s(a, x)<+\infty
$$

and we call this supremum the $\varepsilon$-step extent of $T(a)$.

We define a new 'distance' function on $X \times X$, the $\varepsilon$-step distance, by setting $d_{\varepsilon}(a, b)$ equal to

$$
\inf \left\{\sum_{i=1}^{n} \rho\left(a_{i-1}, a_{i}\right)\right\},
$$

where the infimum is taken over all $a_{0}, a_{1}, \ldots, a_{n} \in X, a_{0}=a, a_{n}=b$ such that $\rho\left(a_{i-1}, a_{i}\right) \leq \varepsilon$, and $a, b \in X$. This has all the properties of a metric, except that its value may be $+\infty$. (One may obtain a proper metric by forming $\arctan \circ d_{\varepsilon}$.) The distance $d_{\varepsilon}$ is a proper metric when restricted to any particular $\varepsilon$-step territory $T(a)$. In general, $d_{\varepsilon}(a, b)$ is at least as large as the original $\rho(a, b)$, but $d_{\varepsilon}(a, b)$ coincides with $\rho(a, b)$ whenever $\rho(a, b) \leq \varepsilon$, and hence $d_{\varepsilon}$ induces the same topology as $\rho$ on $T$, and moreover a function $f: X \rightarrow \mathbb{R}$ is $\rho$-uniformly-continuous if and only if it is $d_{\varepsilon}$-uniformly-continuous. Indeed its $\rho$-modulus of continuity coincides with its $d_{\varepsilon}$-modulus of continuity when the argument is less than or equal to $\varepsilon$.

One readily checks that a territory $T$ is $\varepsilon$-step-bounded if and only if its $d_{\varepsilon}$-diameter is finite. Moreover, its $\varepsilon$-step extent lies between

$$
\frac{d_{\varepsilon}-\operatorname{diam}(T)}{\varepsilon} \quad \text { and } \quad 2+\frac{2 d_{\varepsilon}-\operatorname{diam}(T)}{\varepsilon} .
$$

We now state the main result.

Theorem 2.1. Let $(X, \rho)$ be a metric space. Then the following are equivalent:

(1) Each uniformly-continuous function $f: X \rightarrow \mathbb{R}$ is bounded.

(2) For each $\varepsilon>0, X$ has only a finite number of $\varepsilon$-step territories, and each territory is $\varepsilon$-step-bounded.

Proof. (1) $\Rightarrow$ (2): Suppose (1). Fix $\varepsilon>0$.

Suppose that $X$ has infinitely-many $\varepsilon$-step-territories. Let $T_{n}$ (for $n=1,2,3, \ldots)$ be distinct territories. Then the function $f$, defined by

$$
f(x)= \begin{cases}n & , \quad x \in T_{n}, \\ 0 & , \quad x \in X \sim \bigcup_{n=1}^{\infty} T_{n},\end{cases}
$$

is uniformly-continuous and unbounded, which is impossible. Thus $X$ has only a finite number of $\varepsilon$-step territories. 
Now suppose that one of the $\varepsilon$-step territories, say $T(a)$, is not $\varepsilon$-step-bounded. Define

$$
g(x)=\left\{\begin{aligned}
d_{\varepsilon}(a, x) \quad, \quad x \in T(a), \\
0, \quad x \in X \sim T(a) .
\end{aligned}\right.
$$

Then $g$ is uniformly-continuous on $X$, and unbounded, contradicting the assumption. Thus each $\varepsilon$-step territory is $\varepsilon$-step-bounded, and (2) holds.

$(2) \Rightarrow(1)$ : Suppose (2), and fix $f: X \rightarrow \mathbb{R}$, uniformly-continuous. Pick $\delta>0$ such that $\omega_{f}(\delta)<1$. With $\varepsilon=\delta$, choose $a_{1}, \ldots, a_{n} \in X$ such that $X=\bigcup_{j=1}^{n} T\left(a_{j}\right)$. Then take $N$ to be the maximum of the $\varepsilon$-step extents of the $T\left(a_{j}\right)$, for $1 \leq j \leq n$. Let $M=\max _{j}\left|f\left(a_{j}\right)\right|$.

For each $x \in X$, there exists $j$ with $x \in T\left(a_{j}\right)$, and then there are $x_{0}=a_{j}, x_{1}, \ldots, x_{m}=x$ belonging to $X$, with $m \leq N$ and $\rho\left(x_{i-1}, x_{i}\right) \leq \varepsilon$. Thus

$$
|f(x)| \leq m+\left|f\left(a_{j}\right)\right| \leq N+M .
$$

Thus $f$ is bounded. This proves (1).

Anthony G. O'Farrell,

Mathematics Department,

NUI, Maynooth,

Co. Kildare,

Ireland

aof@maths.may.ie 